\title{
EQUALIZATION OF OFDM WAVEFORMS WITH INSUFFICIENT CYCLIC PREFIX
}

\author{
David Gregoratti and Xavier Mestre \\ Centre Tecnològic de Telecomunicacions de Catalunya (CTTC/CERCA)
}

\begin{abstract}
In this paper, a simple equalization strategy for OFDM waveforms is proposed that specifically targets the case where the cyclic prefix is insufficient to span the whole channel duration. The proposed architecture can be very efficiently implemented in the frequency domain without the need for complex matrix inversions or iterative estimation procedures. Furthermore, the equalizer is shown to outperform conventional methodologies as the system dimensions grow large. Numerical results confirm the excellent behavior of the proposed strategy even for conventional values of the system size.
\end{abstract}

Index Terms - CP-OFDM, high frequency selectivity, equalization, insufficient cyclic prefix.

\section{INTRODUCTION}

The need to comply with strict requirements in terms of ultrareliability and low latency communications (URLLC) has recently motivated the use of shorter OFDM symbols, which have been widely adopted in the New Radio (NR) standard for the fifth generation (5G) of wireless communication systems. Shortening of the total OFDM symbol period leads to a reduction of the corresponding Cyclic Prefix (CP) length, which in practice may become shorter than the actual channel impulse response length. When this happens, the circulant structure of the received signal is destroyed, and a correct signal equalization requires much more elaborate techniques.

OFDM equalization for highly frequency selective channels has been a hot topic of research during the past two decades, and an abundant number of different solutions have been proposed in the literature under different design criteria. One of the first ideas consists in inserting a partial equalizer in the time domain so that the resulting channel fits into the $\mathrm{CP}$ length [1-3]. Other proposals have focused on direct equalization in the frequency domain, either by inverting the inter-carrier interference matrix [4] or by inserting a multi-tap equalizer at the output of the receive DFT [5]. Unfortunately, all these solutions are quite demanding from the computational point of view, and this has motivated the study of simplified equalization architectures that introduce redundancy in the frequency domain. These techniques try to simplify the equalization process by reducing the transmission rate [6-8] or by exploiting the knowledge of channel state information (CSI) at the transmitter [9]. However, in most applications, CSI is only available at the receiver and an important decrease of the spectral efficiency can hardly be afforded. More realistic alternatives try to mitigate the presence of the channel tail by using decision feedback estimates on the transmitted symbols [10-14] or by forcing a brute-force restoration of the circulant signal structure $[15,16]$.

This work has been supported by the Spanish Government under grant RTI2018-099722-B-I00, and by the Catalan Government under grant 2017SGR-01479.
In this paper we propose an alternative equalization structure that is able to provide very accurate equalization without the need for the introduction of additional redundancy in the signal or the implementation of complex decoding mechanisms at the receiver. The approach works in the frequency domain and is able to cancel out most of the residual inter-symbol and inter-carrier interference without a significant increase in terms of computational complexity.

\section{SIGNAL MODEL}

We consider the classic OFDM signal model with $M$ subcarriers: at time $t \in \mathbb{Z}$, the multicarrier symbol $\mathbf{s}[t] \in \mathbb{C}^{M}$ is converted to the time domain by Inverse Discrete Fourier Transform (IDFT) and, after attaching a Cyclic Prefix (CP) of length $N_{\mathrm{P}}$, is transmitted through a time invariant channel with impulse response $h[n]$, $n=1,2, \ldots, L$, with $L<M$. Note that, as opposed to most OFDM-related literature, we do not require the channel impulse response to be shorter than the CP, i.e. it may be $L>N_{\mathrm{P}}$. We will denote by $N$ the length of the OFDM symbol after prefix insertion, i.e. $N=M+N_{\mathrm{P}}$. The discrete-time signal at the receiver side is the result of the convolution $y[n]=h[n] * x[n]$ where the samples $x[n]$ satisfy the identity

$$
\mathbf{x}[t]=\left[\begin{array}{c}
x[(t-1) N+1] \\
x[(t-1) N+2] \\
\vdots \\
x[t N]
\end{array}\right]=\mathbf{\Psi F}_{M} \mathbf{s}[t]
$$

where matrix $\boldsymbol{\Psi}=\left[\begin{array}{c}\mathbf{0}_{N_{\mathrm{P}} \times\left(M-N_{\mathrm{P}}\right)} \mathbf{I}_{N_{\mathrm{P}}} \\ \mathbf{I}_{M}\end{array}\right]$ inserts the CP, with $\mathbf{0}_{m \times n}$ denoting an $m \times n$ matrix filled with zeros and $\mathbf{I}_{m}$ the identity matrix of size $m$. Also, let $\mathbf{F}_{M}$ denote the Fourier matrix, whose $(k, l)$ th entry is given by $\left[\mathbf{F}_{M}\right]_{k, l}=M^{-1 / 2} \exp (\mathrm{j} 2 \pi(k-1)(l-1) / M)$.

The receiver stacks the signal samples, $y[n]$, and builds the vectors $\mathbf{y}[t]=[y[(t-1) N+1] \quad y[(t-1) N+2] \quad \cdots y[t N]]^{\mathrm{T}}$. Then, by means of matrix $\boldsymbol{\Psi}^{(-1)}=\left[\begin{array}{ll}\mathbf{0}_{M \times N_{\mathrm{P}}} & \mathbf{I}_{M}\end{array}\right]$, the $\mathrm{CP}$ is removed before applying the DFT. The frequency-domain signal at the receiver side is thus given by $\mathbf{z}[t]=\mathbf{F}_{M}^{\mathrm{H}} \mathbf{\Psi}^{(-1)} \mathbf{y}[t]$.

\subsection{Received Signal}

It is proven in the Appendix that the multicarrier symbol $\mathbf{z}[t]$ can be compactly expressed as

$$
\mathbf{z}[t]=\boldsymbol{\Delta}_{H} \mathbf{s}[t]-\frac{1}{M} \mathcal{D}_{\tilde{H}}\left(\Theta^{N_{\mathrm{P}}} \mathbf{s}[t]-\mathbf{s}[t-1]\right)
$$

where $\Theta=\operatorname{diag}\{\exp (-\mathrm{j} 2 \pi(m-1) / M)\}_{m=1, \ldots, M}, \mathcal{D}_{\tilde{H}}$ is an $M \times M$ "distortion matrix" described next, and where we have introduced the diagonal matrix $\boldsymbol{\Delta}_{H}$ with $m$ th diagonal entry equal to $H_{L}\left(\omega_{m}\right)$, that is the channel frequency response $H_{L}(\omega)=\sum_{l=1}^{L} h[l] \mathrm{e}^{-\mathrm{j} \omega(l-1)}$ computed at $\omega=\omega_{m}=\frac{2 \pi}{M}(m-1)$. 
In order to provide an expression for $\mathcal{D}_{\tilde{H}}$, let $\tilde{H}_{L}(\omega)$ denote the frequency response of the tail of the channel impulse response beyond the CP, namely $\tilde{H}_{L}(\omega)=\sum_{l=1}^{L-N_{\mathrm{P}}} h\left[l+N_{\mathrm{P}}\right] \mathrm{e}^{-\mathrm{j} \omega(l-1)}$. Then, the distortion matrix $\mathcal{D}_{\tilde{H}}$ is given by

$$
\left[\mathcal{D}_{\tilde{H}}\right]_{m, n}= \begin{cases}\mathrm{j} \tilde{H}_{L}^{\prime}\left(\omega_{m}\right) & \text { if } m=n \\ \frac{\tilde{H}_{L}\left(\omega_{n}\right)-\tilde{H}_{L}\left(\omega_{m}\right)}{1-\mathrm{e}^{-\mathrm{j} \frac{2 \pi}{M}(m-n)}} & \text { otherwise. }\end{cases}
$$

The signal model in (2) is equivalent to (though more elegant than) the one derived in [18, Appendix B]. Specifically, the current form clearly reveals that, when the channel impulse response is longer than the CP, CP-OFDM transmissions are hampered by both inter-symbol and inter-carrier interference induced by $\mathcal{D}_{\tilde{H}}$ and, thus, the classic one-tap-per-subcarrier equalizer is clearly suboptimal. Interestingly enough, the distortion only depends on the portion of the channel impulse response that exceeds the CP. More specifically, (3) tells us that the distortion associated with a high frequency selective channel crucially depends on the variations of the tail of the channel across the frequency domain. Besides, the first part of the channel impulse response, which falls within the $\mathrm{CP}$, plays no role in the signal distortion expression.

\section{CONVENTIONAL AND PROPOSED EQUALIZERS}

Having established a closed form expression for the received signal under strong frequency selectivity, we focus now on the study of equalization procedures that try to recover the transmitted symbols $\mathbf{s}[t]$ from $\mathbf{z}[t]$. More specifically, this section analyzes the behavior of the conventional one-tap-per-subcarrier equalizer and proposes a novel frequency-domain equalization scheme that aims at canceling out the distortion term in (2). The main idea follows the asymptotic reasoning in [17] and establishes that, under some mild conditions on the channel response, the distortion terms can be made asymptotically small by letting the system parameters $\left(M, N_{\mathrm{P}}, L\right)$ grow large. We will assume that the receiver has perfect knowledge of the channel impulse response, which could in principle be obtained according to some other estimation procedure.

\subsection{Conventional One-Tap-per-Subcarrier Equalization}

We first study the behavior of the classic one-tap zero-forcing equalizer, which estimates the transmitted symbols as $\hat{\mathbf{s}}[t]=\boldsymbol{\Delta}_{H}^{-1} \mathbf{z}[t]$. Assuming that the transmitted symbols are generated as independent (across both time and frequency) and identically distributed random variables with zero mean and variance $P$, the per-subcarrier distortion power matrix $\hat{\boldsymbol{\epsilon}}$ can be computed as

$$
\hat{\boldsymbol{\epsilon}} \doteq \mathbb{E}\left[(\mathbf{s}[t]-\hat{\mathbf{s}}[t])(\mathbf{s}[t]-\hat{\mathbf{s}}[t])^{\mathrm{H}}\right]=2 P \mathcal{R}_{\tilde{H}} \mathcal{R}_{\tilde{H}}^{\mathrm{H}}
$$

where we have defined the normalized distortion matrix

$$
\mathcal{R}_{\tilde{H}}=\frac{1}{M} \Delta_{H}^{-1} \mathcal{D}_{\tilde{H}} .
$$

Now, it is clear that if the channel is sufficiently well behaved (in the sense that will be clarified below), the distortion term $\hat{\boldsymbol{\epsilon}}$ could be made as small as desired by allowing $M$ and $N_{\mathrm{P}}$ to grow without bound. In fact, if the channel order $L$ is kept constant, the distortion $\hat{\boldsymbol{\epsilon}}$ will be zero as soon as $N_{\mathrm{P}}>L$ (because $\tilde{H}_{L}$ becomes identically zero). Here, we consider a more general condition whereby $L$ is also allowed to grow with $N_{\mathrm{P}}$, so that their quotient converges to a constant that is generally larger than one. We summarize our working assumptions as:

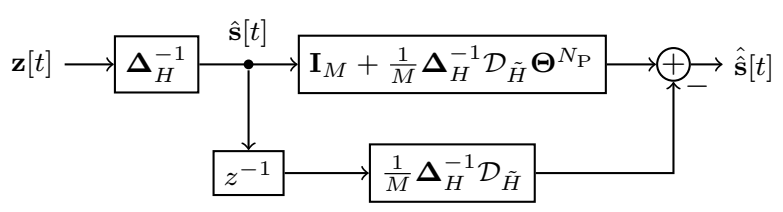

Fig. 1. Proposed frequency-domain equalizer.

(As1) The quantities $M, N_{\mathrm{P}}, L$ converge to infinity at the same rate, so that the quotient between any of these two variables converges to a positive constant.

(As2) The sequence of functions $\left|H_{L}(\omega)\right|$ is bounded away from zero uniformly in $L$, that is $\inf _{L} \inf _{\omega}\left|H_{L}(\omega)\right|>0$.

(As3) There exists a positive number $\ell>0$ such that $|h[n]| \leq$ $n^{-(\ell+\delta+1)}$ for some $\delta>0$ and all $n$ sufficiently large.

Assumption (As1) is a mere technicality that establishes the asymptotic behavior on which our error bounds make sense from the mathematical point of view, whereas assumption (As2) guarantees that the channel is invertible for any value of $L$. On the other hand, assumption (As3) quantifies the channel regularity conditions that guarantee that the error distortion terms decay to zero at a sufficiently high speed. Observe that (As3) directly implies that the total and tail channel responses $H_{L}(\omega), \tilde{H}_{L}(\omega)$ are well defined and continuous in frequency when $L \rightarrow \infty$ (the sequence of channel taps is absolutely summable). We can therefore define $H(\omega) \doteq \lim _{L \rightarrow \infty} H_{L}(\omega)$.

Observe that one can always increase the decay rate of the channel tap coefficients in (As3) by forcing $H(\omega)$ to be sufficiently smooth in the frequency domain. For example, if we guarantee that $H(\omega)$ is $R+1$ times continuously differentiable on the set $[0,2 \pi]_{0 \sim 2 \pi}$ for some $R>0$ (with the canonical identification of the two end points), we will have $|h[n]| \leq K n^{-(R+2)}$ for some positive constant $K$, so that (As3) holds with $\ell=R$ and $\delta=1$.

The following result, proven in the Appendix, will be fundamental in the characterization of the different distortion error terms at the output of the equalizer.

Lemma 1 Under (As1) - (As3), the spectral norm of the matrix $\mathcal{R}_{\tilde{H}}$ decays to zero as $\left\|\mathcal{R}_{\tilde{H}}\right\|_{\infty}=O\left(M^{-\ell}\right)$.

An immediate consequence of Lemma 1 is the fact that $\|\hat{\boldsymbol{\epsilon}}\|_{\infty}=$ $O\left(M^{-2 \ell}\right)$. Since the diagonal entries of a matrix are absolutely bounded by its spectral norm, this directly implies that the distortion power measured at each subcarrier output of the conventional onetap-per-subcarrier equalizer decays to zero as $O\left(M^{-2 \ell}\right)$. By going back to the original received signal in (2), one can alternatively say that the distortion term (second term on the right hand side of that equation) will essentially decay to zero at rate $O\left(M^{-\ell}\right)$, as opposed to the first term that will stay bounded away from zero. In the following subsection, we propose a more sophisticated equalizer that essentially synthesizes the $O\left(M^{-\ell}\right)$ error term and cancels it out from the received signal. The result will lead to a residual distortion power that will decay to zero at a much faster rate, i.e. $O\left(M^{-4 \ell}\right)$.

\subsection{Proposed Equalizer}

The simple structure of (2) suggests that the distortion term can be approximately reconstructed from $\hat{\mathbf{s}}[t]$. As a consequence, a natural equalization scheme is the one depicted in Fig. 1, with

$$
\hat{\mathbf{s}}[t]=\left(\mathbf{I}_{M}+\frac{1}{M} \boldsymbol{\Delta}_{H}^{-1} \mathcal{D}_{\tilde{H}} \Theta^{N_{\mathrm{P}}}\right) \hat{\mathbf{s}}[t]-\frac{1}{M} \boldsymbol{\Delta}_{H}^{-1} \mathcal{D}_{\tilde{H}} \hat{\mathbf{s}}[t-1] .
$$


From the definition of $\hat{\mathbf{s}}[t]$ and $\mathbf{z}[t]$, and using (4), one can easily derive the error vector

$$
\begin{aligned}
\mathbf{s}[t]-\hat{\hat{\mathbf{s}}}[t] & =\left(\mathcal{R}_{\tilde{H}} \boldsymbol{\Theta}^{N_{\mathrm{P}}}\right)^{2} \mathbf{s}[t] \\
& -\mathcal{R}_{\tilde{H}}\left(\boldsymbol{\Theta}^{N_{\mathrm{P}}} \mathcal{R}_{\tilde{H}}+\mathcal{R}_{\tilde{H}} \boldsymbol{\Theta}^{N_{\mathrm{P}}}\right) \mathbf{s}[t-1]+\mathcal{R}_{\tilde{H}}^{2} \mathbf{s}[t-2]
\end{aligned}
$$

and the resulting distortion power matrix can be written as

$$
\begin{aligned}
\hat{\hat{\boldsymbol{\epsilon}}} & \doteq \mathbb{E}\left[(\mathbf{s}[t]-\hat{\hat{\mathbf{s}}}[t])(\mathbf{s}[t]-\hat{\hat{\mathbf{s}}}[t])^{\mathrm{H}}\right] \\
& =2 P \mathcal{R}_{\tilde{H}} \boldsymbol{\Theta}^{N_{\mathrm{P}}} \mathcal{R}_{\tilde{H}} \mathcal{R}_{\tilde{H}}^{\mathrm{H}} \boldsymbol{\Theta}^{-N_{\mathrm{P}}} \mathcal{R}_{\tilde{H}}^{\mathrm{H}}+2 P \mathcal{R}_{\tilde{H}}^{2}\left(\mathcal{R}_{\tilde{H}}^{\mathrm{H}}\right)^{2} \\
& +P \mathcal{R}_{\tilde{H}}\left(\mathcal{R}_{\tilde{H}} \boldsymbol{\Theta}^{N_{\mathrm{P}}} \mathcal{R}_{\tilde{H}}^{\mathrm{H}} \boldsymbol{\Theta}^{-N_{\mathrm{P}}}+\boldsymbol{\Theta}^{N_{\mathrm{P}}} \mathcal{R}_{\tilde{H}} \boldsymbol{\Theta}^{-N_{\mathrm{P}}} \mathcal{R}_{\tilde{H}}^{\mathrm{H}}\right) \mathcal{R}_{\tilde{H}}^{\mathrm{H}} .
\end{aligned}
$$

Now, a direct application of Lemma 1 shows that the distortion power at the output of the proposed equalizer decays to zero as $\|\hat{\hat{\boldsymbol{\epsilon}}}\|_{\infty}=O\left(M^{-4 \ell}\right)$, that is much faster than the original onetap-per-subcarrier equalizer. We will show in Section 4 that this will translate into a much better performance even for relatively low values of the system parameters $L, M, N_{\mathrm{P}}$.

The computational complexity of the proposed equalizer is extremely low. The proposed architecture does not need matrix inversions and provides a direct equalized signal without the need of iterative loops. Most of the operations can be performed at the persubcarrier level, the only exception being the multiplication by the $M \times M$ matrix $\mathcal{D}_{\tilde{H}}$. However, it can readily be seen from (3) that one can decompose $\mathcal{D}_{\tilde{H}}$ by a diagonal matrix containing the derivatives of the tail response plus a matrix of the form $\left(\mathbf{1}_{M} \mathbf{h}^{\mathrm{T}}-\mathbf{h} \mathbf{1}_{M}^{\mathrm{T}}\right) \odot \mathbf{M}$ where $\mathbf{h}$ is a column vector containing the tail frequency response, $\odot$ denotes the element-wise product, $\mathbf{1}_{M}$ is an $M \times 1$ all-one column vector and $\mathbf{M}$ is a certain circulant matrix. Moreover, it can be shown that for any column vector $\mathbf{x}$, we have $\left(\mathbf{1}_{M} \mathbf{h}^{\mathrm{T}} \odot \mathbf{M}\right) \mathbf{x}=$ $\mathbf{M}(\mathbf{h} \odot \mathbf{x})$ and $\left(\mathbf{h} \mathbf{1}_{M}^{\mathrm{T}} \odot \mathbf{M}\right) \mathbf{x}=\mathbf{h} \odot \mathbf{M x}$. Therefore, we can implement the multiplication by $\mathcal{D}_{\tilde{H}}$ with simple per-subcarrier operations plus multiplications by a circulant matrix, which can be efficiently carried out with the aid of the FFT in $O(M \log M)$ operations.

With similar tools as above, and assuming that the information signal $\mathbf{y}[t]$ is contaminated by an additive white Gaussian noise vector $\mathbf{n}[t] \sim \mathcal{N}_{\mathbb{C}}\left(\mathbf{0}_{N}, \sigma^{2} \mathbf{I}_{N}\right)$, one can prove that the output noise covariance matrix is $\mathbb{E}\left[\hat{\boldsymbol{\eta}}[t] \hat{\boldsymbol{\eta}}^{\mathrm{H}}[t]\right]=\sigma^{2}\left(\boldsymbol{\Delta}_{H} \boldsymbol{\Delta}_{H}^{\mathrm{H}}\right)^{-1}$ for the classic equalizer and

$$
\begin{aligned}
\mathbb{E}[\hat{\boldsymbol{\eta}}[t] & \left.\hat{\hat{\boldsymbol{\eta}}}^{\mathrm{H}}[t]\right]=\sigma^{2} \mathcal{R}_{\tilde{H}} \boldsymbol{\Delta}_{H}^{-1} \boldsymbol{\Delta}_{H}^{-\mathrm{H}} \mathcal{R}_{\tilde{H}}^{\mathrm{H}} \\
& +\sigma^{2}\left(\mathbf{I}_{M}+\mathcal{R}_{\tilde{H}} \boldsymbol{\Theta}^{N_{\mathrm{P}}}\right) \boldsymbol{\Delta}_{H}^{-1} \boldsymbol{\Delta}_{H}^{-\mathrm{H}}\left(\mathbf{I}_{M}+\boldsymbol{\Theta}^{-N_{\mathrm{P}}} \mathcal{R}_{\tilde{H}}^{\mathrm{H}}\right) .
\end{aligned}
$$

for the proposed one.

\section{NUMERICAL RESULTS}

To assess the results presented in this paper, we consider a system with $M=512$ subcarriers, and we fix the CP length to $N_{\mathrm{P}}=36$ (around the $7 \%$ of the symbol length, as commonly used in the $4 \mathrm{G}$ and 5G standards). The channel model assumes independent taps with an exponentially decreasing power profile, that is $\mathbb{E}\left[|h[l]|^{2}\right]=$ $\exp (-(l-1) / \tau)$, which meets assumptions (As1)-(As3). By selecting $\tau=10$ the channel impulse response becomes negligible (taps are at least $30 \mathrm{~dB}$ below the first one) after approximately 80 taps, even though we let $L=M=512$.

For a given channel realization, we report in Fig. 2 the comparison between the empirical distortion power (averaged over 1000 symbols) and the expressions derived in Section 3. Besides the perfect match, we also observe a remarkable gain of approximately $15 \mathrm{~dB}$ of the proposed equalizer over the classic one-tap solution.

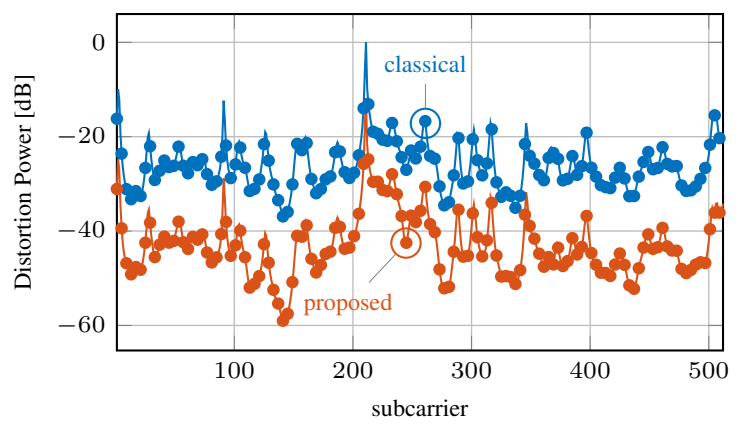

Fig. 2. Comparison of the distortion power for the classic and proposed equalizers, both simulated (continuous line) and theoretic (markers).

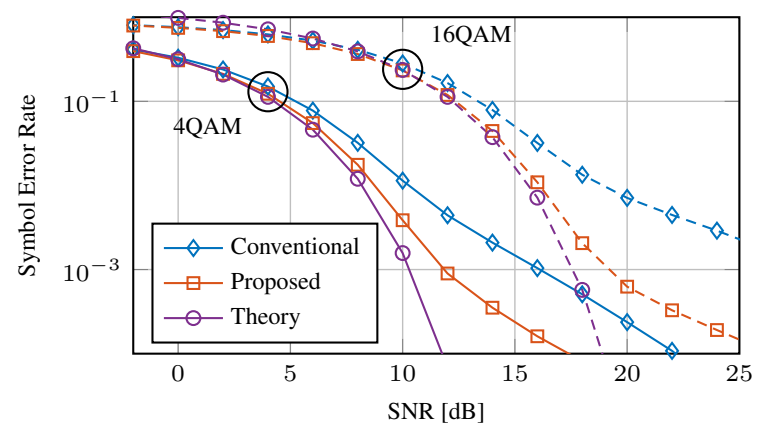

Fig. 3. Symbol error rate for $4 \mathrm{QAM}$ and 16QAM symbols.

As an additional evidence of the superiority of the proposed equalizer over the classical one-tap-per-subcarrier one, Fig. 3 reports the empirical Symbol Error Rate (SER) curves as a function of SNR $=P H_{L}^{2}\left(\omega_{m}\right) \sigma^{2}$ for 4QAM and 16QAM constellations. These curves represent the Monte-Carlo average over 1000 realizations of a channel with the characteristics outlined above. Also, each realization corresponds to the transmission of a batch of 100 QAM multicarrier symbols. We notice that the proposed equalizer loses less than $2 \mathrm{~dB}$ with respect to the theoretic QAM curve up to $\mathrm{SER}=10^{-3}$ and that it clearly outperforms the classic solution. This is especially true in the 16QAM case, where the one-tap equalizer curve changes its slope towards an error floor just below SER $=10^{-2}$.

\section{CONCLUSIONS}

A novel equalization strategy for OFDM waveforms in the presence of insufficient cyclic prefix has been presented. The proposed algorithm works in the frequency domain by sequentially canceling the error terms caused by the excess channel impulse response. The strategy can be efficiently implemented without the need for complex matrix inversions or iterative procedures. An asymptotic characterization of the output error has been provided, showing that the distortion power decays substantially faster than the conventional equalizer when the system dimensions increase without bound. Simulation results demonstrate the good performance of the equalizer, which is able to provide substantial gains even for relatively low system dimensions. 


$$
\begin{aligned}
& \mathbf{\Psi}^{(-1)} \mathbf{y}_{l}[t]=\left[\begin{array}{c}
{\left[\mathbf{F}_{M}\right]_{\left(M+N_{\mathrm{P}}-l+2\right): M}^{\mathrm{T}}} \\
\mathbf{0}_{\left(M+N_{\mathrm{P}}-l+1\right) \times M}
\end{array}\right] \mathbf{s}[t-1]+\left[\begin{array}{c}
\mathbf{0}_{\left(l-N_{\mathrm{P}}-1\right) \times M} \\
{\left[\mathbf{F}_{M}\right]_{\left[M-N_{\mathrm{P}}+1: M\right]}^{\mathrm{T}}} \\
{\left[\mathbf{F}_{M}\right]_{1: M-l+1}^{\mathrm{T}}}
\end{array}\right] \mathbf{s}[t] \\
& =\mathcal{C}_{M}^{l-N_{\mathrm{P}}-1}\left[\begin{array}{cc}
\mathbf{0}_{\left(M+N_{\mathrm{P}}-l+1\right) \times M} \\
\mathbf{0}_{\left(l-N_{\mathrm{P}}-1\right) \times\left(M+N_{\mathrm{P}}-l+1\right)} & \mathbf{I}_{l-N_{\mathrm{P}}-1}
\end{array}\right] \mathbf{F}_{M} \mathbf{s}[t-1]+\mathcal{C}_{M}^{l-1}\left[\begin{array}{cc}
\mathbf{I}_{M-l+1} & \mathbf{0}_{(M-l+1) \times(l-1)} \\
\mathbf{0}_{\left(l-N_{\mathrm{P}}-1\right) \times M} \\
\mathbf{0}_{N_{\mathrm{P}} \times\left(M-N_{\mathrm{P}}\right)} & \mathbf{I}_{N_{\mathrm{P}}}
\end{array}\right] \mathbf{F}_{M} \mathbf{s}[t]
\end{aligned}
$$

\section{A. APPENDIX: PROOF OF (2)}

In order to prove (2), we first compute vector $\mathbf{z}_{l}[t]$, that is the multicarrier symbol recovered by the receiver when the channel simply introduces a delay of $(l-1)$ taps. Then, by linearity, vector $\mathbf{z}[t]$ in the general case can be easily computed as $\mathbf{z}[t]=\sum_{l=1}^{L} h[l] \mathbf{z}_{l}[t]$.

By simple inspection, when $h[n]=1$ for $n=l$ and zero elsewhere, one readily sees that $\mathbf{y}_{l}[t]=[x[(t-1) N-l+2] \quad x[(t-$ 1) $N-l+3] \quad \cdots \quad x[t N-l+1]]^{\mathrm{T}}$ (as with $\mathbf{z}[t]$, we introduce the subscript $l$ to $\mathbf{y}[t]$ to stress its dependence on the delay). It also follows that

$$
\mathbf{\Psi}^{(-1)} \mathbf{y}_{l}[t]=\left[\begin{array}{c}
x\left[(t-1) N+N_{\mathrm{P}}-l+2\right] \\
x\left[(t-1) N+N_{\mathrm{P}}-l+3\right] \\
\vdots \\
x[t N-l+1]
\end{array}\right]
$$

From (1), the entries of the above vector can be written as

$$
x[(t-1) N+n]= \begin{cases}\mathbf{f}_{M-N_{\mathrm{P}}+n}^{\mathrm{T}} \mathbf{s}[t] & \text { if } 1 \leq n \leq N_{\mathrm{P}} \\ \mathbf{f}_{n-N_{\mathrm{P}}}^{\mathrm{T}} \mathbf{s}[t] & \text { if } N_{\mathrm{P}}+1 \leq n \leq N\end{cases}
$$

with $\mathbf{f}_{n}$ the $n$th column of $\mathbf{F}_{M}$. We now differentiate two separate cases regarding the delay index $l$.

Case $1 \leq l \leq N_{\mathrm{P}}+1 \quad$ When the delay is shorter than the CP, (5) reads

$$
\mathbf{\Psi}^{(-1)} \mathbf{y}_{l}[t]=\left[\begin{array}{c}
{\left[\mathbf{F}_{M}\right]_{M-l+2: M}^{\mathrm{T}}} \\
{\left[\mathbf{F}_{M}\right]_{1: M-l+1}^{\mathrm{T}}}
\end{array}\right] \mathbf{s}[t]=\mathcal{C}_{M}^{l-1} \mathbf{F}_{M} \mathbf{s}[t]
$$

where $\left[\mathbf{F}_{M}\right]_{n_{1}: n_{2}}$ is the submatrix built from columns $n_{1}$ to $n_{2}$ of $\mathbf{F}_{M}$ and $\mathcal{C}_{M}$ is the unitary circulant matrix, that is a circulant matrix with first row $\left[\begin{array}{llll}0 & \cdots & 0 & 1\end{array}\right]$. As a result

$$
\mathbf{z}_{l}[t]=\mathbf{F}_{M}^{\mathrm{H}} \mathcal{C}_{M}^{l-1} \mathbf{F}_{M} \mathbf{s}[t]=\mathbf{\Theta}^{l-1} \mathbf{s}[t]
$$

where we used the fact that a rotation in the frequency domain corresponds to phase modulation in the time domain, namely $\mathbf{F}_{M}^{\mathrm{H}} \mathcal{C}_{M}^{l-1}=$ $\Theta^{l-1} \mathbf{F}_{M}^{\mathrm{H}}$, with $\Theta$ the diagonal matrix defined in (2). As expected from classical OFDM results, when the delay is shorter than the $\mathrm{CP}$, the transmitted multicarrier symbol is perfectly recovered at the receiver side apart from a diagonal matrix with the channel frequency response.

Case $l>N_{\mathrm{P}}+1$ When the delay is longer than the CP, we observe that the top $l-N_{\mathrm{P}}+1$ entries in (5) depend on the previous transmitted symbol, $\mathbf{s}[t-1]$, as opposed to the current one. Then, the received signal after removing the $\mathrm{CP}$ is given by (7) at the top of this page.
Let us introduce the family of complex $M \times M$ matrices

$$
\mathbf{D}(k)=M \mathbf{F}_{M}^{\mathrm{H}}\left[\begin{array}{cc}
\mathbf{I}_{k} & \mathbf{0}_{k \times(M-k)} \\
\mathbf{0}_{(M-k) \times M}
\end{array}\right] \mathbf{F}_{M}
$$

for all $k=0,1, \ldots, M$. After trivial algebra, the matrix entries can be shown to be

$[\mathbf{D}(k)]_{m, n}= \begin{cases}k & \text { if } m=n \\ \mathrm{e}^{-\mathrm{j} \frac{\pi}{M}(k-1)(m-n)} \frac{\sin \left(\frac{\pi}{M} k(m-n)\right)}{\sin \left(\frac{\pi}{M}(m-n)\right)} & \text { otherwise }\end{cases}$

(note the connection to the Dirichlet kernel).

Then, the receiver multicarrier symbol can be written as

$$
\begin{aligned}
\mathbf{z}_{l}[t]=\mathbf{D}(l- & \left.N_{\mathrm{P}}-1\right) \boldsymbol{\Theta}^{l-N_{\mathrm{P}}-1} \mathbf{s}[t-1] \\
& +\left(\boldsymbol{\Theta}^{l-1} \mathbf{I}_{M}-\mathbf{D}\left(l-N_{\mathrm{P}}-1\right) \boldsymbol{\Theta}^{l-1}\right) \mathbf{s}[t] .
\end{aligned}
$$

To conclude the proof, we now need to combine (6) and (9) into the general-case recovered multicarrier symbol, namely

$$
\begin{gathered}
\mathbf{z}[t]=\left(\sum_{l=1}^{L} h[l] \mathbf{\Theta}^{l-1}\right) \mathbf{s}[t] \\
-\left(\sum_{l=N_{\mathrm{P}}+2}^{L} h[l] \mathbf{D}\left(l-N_{\mathrm{P}}-1\right) \mathbf{\Theta}^{l-1}\right)\left(\mathbf{s}[t]-\mathbf{\Theta}^{-N_{\mathrm{P}}} \mathbf{s}[t-1]\right) .
\end{gathered}
$$

The form in (2) is a direct consequence of the definitions of $H_{L}(\omega)$, $\tilde{H}_{L}(\omega)$ and $\mathbf{D}(l)$, together with the fact that $\mathbf{D}(0)=\mathbf{0}_{M}$. Specifically, $\boldsymbol{\Delta}_{H}=\sum_{l=1}^{L} h[l] \Theta^{l-1}$ and

$$
\mathcal{D}_{\tilde{H}}=\sum_{l=1}^{L-N_{\mathrm{P}}} h\left[l+N_{\mathrm{P}}\right] \mathbf{D}(l-1) \boldsymbol{\Theta}^{l-1} \text {. }
$$

\section{B. APPENDIX: PROOF OF LEMMA 1}

From the submultiplicative nature of the spectral norm, we readily see that

$$
\left\|\mathcal{R}_{\tilde{H}}\right\|_{\infty} \leq M^{-1}\left\|\boldsymbol{\Delta}_{H}^{-1}\right\|_{\infty}\left\|\mathcal{D}_{\tilde{H}}\right\|_{\infty}
$$

where $\sup \left\|\boldsymbol{\Delta}_{H}^{-1}\right\|_{\infty}<\infty$ by (As2). On the other hand, using the expression of $\mathcal{D}_{\tilde{H}}$ in (10), we see that

$$
\left\|\mathcal{D}_{\tilde{H}}\right\|_{\infty} \leq \frac{1}{M} \sum_{l=1}^{L-N_{\mathrm{P}}}\left|h\left[l+N_{\mathrm{P}}\right]\right|\|\mathbf{D}(l-1)\|_{\infty}
$$

where $\mathbf{D}(n)$ is defined in (8). In particular, from the expression of $\mathbf{D}(n)$ we readily see that it has spectral norm equal to $M$, so that

$$
\left\|\mathcal{D}_{\tilde{H}}\right\|_{\infty} \leq \sum_{l=1}^{\infty}\left|h\left[l+N_{\mathrm{P}}\right]\right|
$$

and the result follows directly from (As3). 


\section{REFERENCES}

[1] N. Al-Dhahir and J. M. Cioffi, "Optimum finite-length equalization for multicarrier transceivers," IEEE Trans. Commun., vol. 44, no. 1, pp. 56-64, Jan. 1996.

[2] G. Arslan, B. L. Evans, and S. Kiaei, "Optimum channel shortening for discrete multitone transceivers," in IEEE International Conference on Acoustics, Speech, and Signal Processing, June 2000, vol. 5, pp. 2965-2968 vol.5.

[3] S. Ma and T. Ng, "Semi-blind time-domain equalization for MIMO-OFDM systems," IEEE Trans. Veh. Technol., vol. 57, no. 4, pp. 2219-2227, July 2008.

[4] M. D. Nisar, W. Utschick, H. Nottensteiner, and T. Hindelang, "On channel estimation and equalization of OFDM systems with insufficient cyclic prefix," in IEEE Vehicular Technology Conference (VTC Spring), Apr. 2007, pp. 1445-1449.

[5] G. Leus and M. Moonen, "Per-tone equalization for MIMO OFDM systems," IEEE Trans. Signal Process., vol. 51, no. 11, pp. 2965-2975, Nov. 2003.

[6] Y. Jin and X. Xia, "A robust precoder design based on channel statistics for MIMO-OFDM systems with insufficient cyclic prefix," IEEE Trans. Commun., vol. 62, no. 4, pp. 1249-1257, Apr. 2014.

[7] Cheol-Jin Park and Gi-Hong Im, "Efficient DMT/OFDM transmission with insufficient cyclic prefix," IEEE Commun. Lett., vol. 8, no. 9, pp. 576-578, Sept. 2004.

[8] Y. Jin and X. Xia, "An interference nulling based channel independent precoding for MIMO-OFDM systems with insufficient cyclic prefix," IEEE Trans. Commun., vol. 61, no. 1, pp. 131-143, Jan. 2013.

[9] Kok-Wui Cheong and J. M. Cioffi, "Precoder for DMT with insufficient cyclic prefix," in IEEE International Conference on Communications, June 1998, vol. 1, pp. 339-343.

[10] S. Ariyavisitakul, N. R. Sollenberger, and L. J. Greenstein, "Tap-selectable decision-feedback equalization," IEEE Trans. Commun., vol. 45, no. 12, pp. 1497-1500, Dec. 1997.

[11] S. Ariyavisitakul and L. J. Greenstein, "Reduced-complexity equalization techniques for broadband wireless channels," IEEE J. Sel. Areas Commun., vol. 15, no. 1, pp. 5-15, Jan. 1997.

[12] O. Barbu, C. N. Manchón, C. Rom, and B. H. Fleury, "Message-passing receiver for OFDM systems over highly delay-dispersive channels," IEEE Trans. Wireless Commun., vol. 16, no. 3, pp. 1564-1578, Mar. 2017.

[13] T. Pham, T. Le-Ngoc, G. Woodward, P. A. Martin, and K. T. Phan, "Equalization for MIMO-OFDM systems with insufficient cyclic prefix," in IEEE Vehicular Technology Conference (VTC Spring), May 2016, pp. 1-5.

[14] T. Pham, T. Le-Ngoc, G. K. Woodward, and P. A. Martin, "Channel estimation and data detection for insufficient cyclic prefix MIMO-OFDM," IEEE Trans. Veh. Technol., vol. 66, no. 6, pp. 4756-4768, June 2017.

[15] Cheol-Jin Park and Gi-Hong Im, "Efficient cyclic prefix reconstruction for coded OFDM systems," IEEE Commun. Lett., vol. 8, no. 5, pp. 274-276, May 2004.

[16] Dukhyun Kim and G. L. Stuber, "Residual ISI cancellation for OFDM with applications to HDTV broadcasting," IEEE J. Sel. Areas Commun., vol. 16, no. 8, pp. 1590-1599, Oct. 1998.
[17] Xavier Mestre and David Gregoratti, "Parallelized structures for MIMO FBMC under strong channel frequency selectivity," IEEE Trans. Signal Process., vol. 64, no. 5, pp. 1200-1215, Mar. 2016.

[18] David Gregoratti and Xavier Mestre, "Uplink FBMC/OQAMbased multiple access channel: Distortion analysis under strong frequency selectivity," IEEE Trans. Signal Process., vol. 64, no. 16, pp. 4260-4272, Aug. 2016. 